NASA Contractor Report 194436

AIAA-93-3812

203538

\title{
A Comparison of Two Multi-Variable Integrator Windup Protection Schemes
}

Duane Mattern

Sverdrup Technology, Inc.

Lewis Research Center Group

Brook Park, Ohio

Prepared for the

1993 AIAA Guidance, Navigation, and Control Conference

sponsored by the American Institute of Aeronautics and Astronautics

Monterey, California, August 9-11, 1993

(NASA-CR-194436) A COMPARISON OF

TWO MIJLTI-VARIABLE INTEGRATOR

WINDUP PROTECTION SCHEMES Final

Report (Sverdrup Technology) $11 \mathrm{p}$
N94-23590

Unclas 


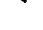




\title{
A Comparison of Two Multi-Variable Integrator Windup Protection Schemes
}

\author{
Duane Mattern \\ Sverdrup Technology, Inc. \\ Lewis Research Center Group \\ Brook Park, Ohio 44142
}

\begin{abstract}
$\underline{\text { Abstract }}$
Two methods are examined for limit and integrator wind-up protection for multi-input, multioutput linear controllers subject to actuator constraints. The methods begin with an existing linear controller that satisfies the specifications for the nominal, small perturbation, linear model of the plant. The controllers are formulated to include an additional contribution to the state derivative calculations. The first method to be examined is the multi-variable version of the singleinput. single-output. high gain, Conventional AntiWindup (CAW) scheme. Except for the actuator limits, the CAW scheme is linear. The second scheme to be examined, denoted the Modified Anti-Windup (MAW) scheme. uses a scalar to modify the magnitude of the controller output vector while maintaining the vector direction. The calculation of the scalar modifier is a nonlinear function of the controller outputs and the actuator limits. In both cases the constrained actuator is tracked. These two integrator windup protection methods are demonstrated on a turbofan engine control system with five measurements, four control variables, and four actuators. The closed-loop responses of the two schemes are compared and contrasted during limit operation. The issue of maintaining the direction of the controller output vector using the Modified Anti-Windup scheme is discussed and the advantages and disadvantages of both of the IWP methods are presented.
\end{abstract}

\section{Nomenclature}

Perturbed vectors

$u_{c}{ }^{\mathrm{L}}$ - controller output vector, subject to constraints

$u_{c} \quad$ controller output vector, (nu $\left.\times 1\right)$

$u_{d} \quad$ desired controller output, (nu $x 1$ )

$u_{1} \quad$ actuator constraints, (nu $x$ 1)

$x_{c} \quad$ controller state vector, $\left(n x_{r} \times 1\right)$

$\mathrm{x} \quad$ state vector, $(\mathrm{n} \times \mathrm{x} 1)$

$y \quad$ vector of measured variables, (ny $x 1$ )

$z \quad$ vector of controlled variable, $(n z \times 1)$

\author{
Matrices \\ A system matrix of appropriate size \\ B input matrix of appropriate size \\ C output matrix of appropriate size \\ D feedthrough matrix of appropriate size \\ L diagonal limit indicator matrix, (nu $x$ nu) \\ $\Lambda \quad$ CAW Scheme gain matrix, $\left(n x_{c} \times n u\right)$ \\ Scalars \\ a variable multiplier for the MAW scheme \\ $\beta$ constant feedback gain for the MAW scheme \\ $\eta \quad$ constant feedback gain for the CAW scheme \\ WF36 fuel flow, $\mathrm{lbm} / \mathrm{hr}$ \\ A8 aft nozzle area, square inches \\ Eta ejector butterfly valve angle. degrees \\ A78 ventral nozzle area, square inches \\ FG9 aft nozzle thrust. Ibf \\ FGE ejector thrust, lbf \\ FGV ventral nozzle thrust, Ibf \\ N2 fan compressor rotor speed, rpm \\ N25 core compressor rotor speed, rpm \\ Subscripts \\ c controller variable \\ d desired value \\ i i'th scalar variable in a vector list \\ 1 limit value \\ u corresponding to the input vector \\ $\mathrm{x}$ corresponding to the state vector
}

\section{Introduction}

Research in integrated flight and propulsion control performed at the Advanced Control Technology Branch of the NASA Lewis Research Center has resulted in an Integrated Method for Propulsion and Airframe Control (IMPAC) design [1]. To evaluate this method, an example control system is being designed for a model of a Short Take-Off and Vertical Landing (STOVL) aircraft during transition flight. The steps of the IMPAC design are shown in Figure 1. Step five of the method is the nonlinear control design for the various subsystems. The nonlinear control design includes the addition of washout filters and trim logic, gain scheduling, limit logic, and limit and integrator 
windup protection (IWP). Preliminary evaluations of the NASA Lewis linear integrated control system for large command inputs have indicated the need for limit and integrator windup protection on the propulsion and aircraft subsystems. Integrator windup protection is common in control implementations, and schemes for single-input, single-output (SISO) systems have been discussed in the literature. General, multi-input, multioutput (MIMO) integrator wind-up protection methods have not received a great deal of attention in the literature, until recently. This paper examines and compares two of the available multi-variable integrator windup protection schemes for linear control systems with constraints imposed upon the range of the actuators.

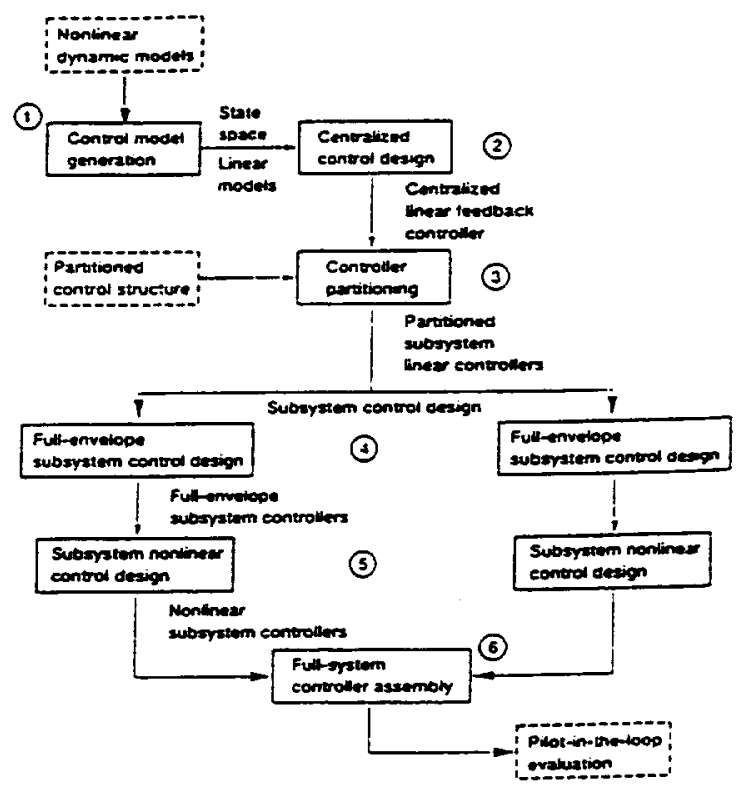

Figure 1 IMPAC Methodology Flowchart

In the following, the integrator wind-up and limit protection problem for general, multi-input, multioutput, linear controllers is presented. Then, the literature on multi-variable integrator windup methods is briefly reviewed. Next, two methods, the Conventional Anti-Windup (CAW) and the Modified Anti-Windup (MAW) schemes are examined in detail. To test these two schemes, an example problem is described. consisting of a linear control system for a linear model of a turbofan engine with actuator range limits. The closed-loop performance of the CAW and the MAW schemes during limit operation are compared. Finally, the advantages and disadvantages of these two IWP methods are discussed.

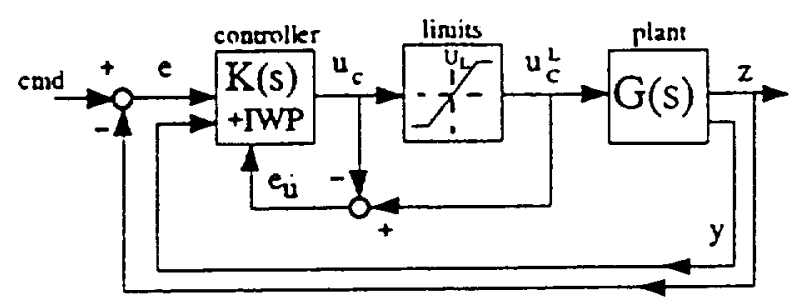

Figure 2 Typical Limit Protection Problem Structure

\section{The Integrator Windup Problem}

All control implementations have the possibility of encountering constraints such as actuator range and rate limits or system safery limits reflected back upon the plant inputs. When limits occur, the control system integrals can wind up if the controller was designed to drive the steady-state errors to zero. The windup appears as an increase in the magnitude of the integrators beyond that which would normally be observed during linear operation for the same inputs. A simple example of integrator windup is an error driven SISO proportional plus integral plus derivative, (PID), control system with a limited actuator. With the actuator constrained, the error can not be driven to zero. As long as the error is nonzero and of constant numerical sign, the integral term in the controller will increase in magnitude. Integrator windup is only unbounded for a constant constraint. Transient encounters with the actuator constraints cause shor duration integrator windup that can lead to oscillations such as limit cycles or delayed responses due to the controller holding the limit value until the integrator "unwinds". A typical structure for the limit protection problem is shown in Figure 2. The structure consists of a plant, $G(s)$, a nomina! linear controller, $K(s)$, and actuator range limits. The linear, time invariant, small perturbation, model of the plant, $G(s)$, is represented in state space format as follows:

$$
\begin{gathered}
\dot{x}=A x+B u \\
{\left[\begin{array}{l}
z \\
y
\end{array}\right]=C x+D u}
\end{gathered}
$$

The linear control system, $K(s)$, satisfies the nominal closed-loop, small perturbation specifications and is represented by the following state-space structure: 


$$
\begin{aligned}
& \dot{x}_{c}=A_{c} x_{c}+B_{c}\left[\begin{array}{l}
e \\
y
\end{array}\right] \\
& u_{c}=C_{c} x_{c}+D_{c}\left[\begin{array}{l}
e \\
y
\end{array}\right]
\end{aligned}
$$

Figure 2 shows that the controller output vector, $u_{c}$, is the input to the limit block. The vector output of the limit block is $u_{c}{ }^{L}$. As long as the magnitude of the $i^{\prime}$ th element of $u_{c}, u_{c}$, is less than the $i$ 'th actuator limit value, $u_{j}$, then $u_{c i}{ }^{2}=u_{f}$, otherwise $u_{c i}{ }^{2}$ is equal to the corresponding actuator limit value, $u_{\mathrm{j}}$. The actuator limit error, defined as $e_{c}=u_{c}{ }^{2}-u_{c}$, is fed back to the IWP scheme. Actuator limits are typically taken into consideration during the selection of the input scale factors and weights that are used in the nominal control design. The actuator limits will be encountered whenever maximum performance is required as the constraints define the maximum system performance. IWP will be required during this limit operation. The specifications on IWP schemes, as discussed in Reference [2], are as follows:

1) An actuator limit represents a constraint in the input space and must be observable.

2) The IWP scheme should not contribute to the nominal control system when the system is unconstrained. This indicates that the IWP scheme should be memoryless.

3) The controller with IWP should track the limited actuator value such that the transition from limit mode to unlimited mode is smooth, (a "bumpless transfer").

4) The IWP scheme should be closed-loop stable for all possible limit combinations.

5) When actuator limits occur, the IWP scheme should maintain system performance. If it is not possible to maintain the closed-loop performance, then the IWP scheme should degrade the system performance gracefully, while maintaining stability. In the following, the actuator limit values are assumed to be known so that the controller outputs can be limited within the control itself, before the actuator command goes to the actuator. Also, the IWP schemes discussed here are memoryless. Guarantees for closed-loop system stability with actuator constraints and guarantees for closed-loop performance for systems with redundant actuators are both current research topic and are outside of the scope of this paper. The literature on multi-input. multi-output limit and integrator windup protection schemes will now be reviewed.

\section{Integrator Windup Protection Schemes}

The literature on limit and integrator windup protection discusses several techniques. For SISO systems, the error that drives the integral error term is typically zeroed when the system encounters a limit. Other SISO schemes [3], have fod back the observed actuator error, $e_{a}$, as done in Figure 2. This error is used to drive the controller to track the limited actuator, putting a limit tracking proportional regulator upon the controller itself. Several versions of this $e_{\mathrm{n}}$ feedback approach have been extended to MIMO systems and they are known, collectively, as the Conventional AntiWindup (CAW) method. In a MIMO system, an encounter with a single limit changes the direction of controller output vector, $u_{c}$. Figure 3 shows such an example for a two dimensional vector during an encounter with the limit for the first actuator such that $u_{\mathrm{rl}}=\mathrm{u}_{\mathrm{L}}$. The scalar approach modifies the magnitude and not the direction of $u_{c}$, where as the CAW scheme truncates the limited actuator value. Some systems are sensitive to changes in the direction of $u_{c}$ and for these systems an actuator limit can result in poor closed-loop performance.

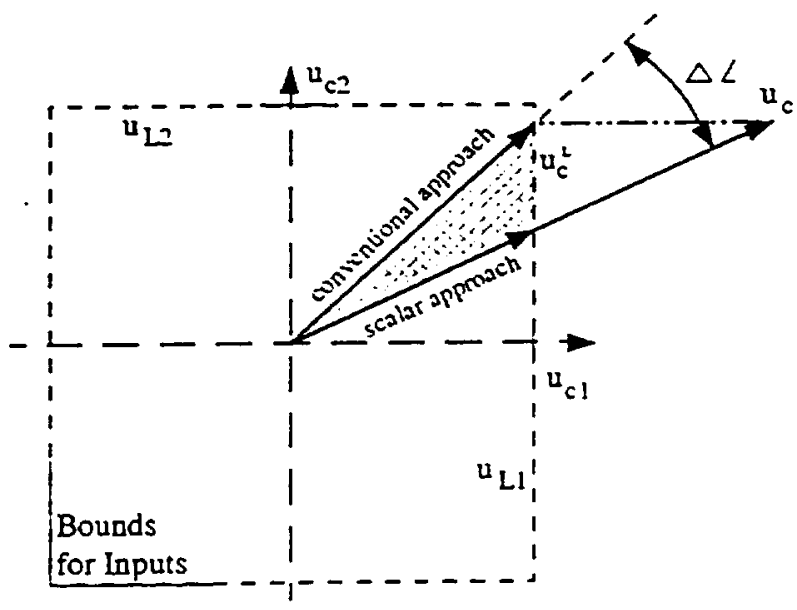

Figure 3 2D Actuator Directionality

Reference [4] addresses this directionality issue for stable plants and square controllers by multiplying the error vector, $e$, by a scalar, $\lambda(t),(0 \leq \lambda \leq 1)$. This "enor govemor" is a function of the boundary of the admissible controller state which can be calculated by reflecting the actuator limits onto the controller states and by predicting bounds for the controller state trajectory. The scalar multiplier prevents the controller from exceeding the actuator limit and zeros the error input to the controller when a saturation is reached. 
The usefulness of this method is limited because it requires the solution to $C_{c} e^{\lambda_{1}} x_{r}=u_{1}$ for the controller state, $x_{r}$ such that any possible combination of the actuator limits, $u$, are not violated in onder to determine the region for the admissible controller state. This problem is formidable, particularly if one considers a controller with time varying limits, or a gain scheduled controller, or nonsymmetric bounds for 4 . Also, the solution to this problem is not unique if there are more controller state variables than controller outputs.

Reference [5] presents an alternative approach to the limit problem using linear robust control design tools. In Reference [5], the saturation function is reformulated as a dead-zone nonlinearity to model the actuator limits as a sector bounded uncertainty. Then, linear multi-variable control design methods guarantee the system closed-loop robust stability for specific levels of this "uncertainty". Adding actuator limit uncertainty to all of the plant inputs yields a design that is similar to one obtained by increasing the weights on all inputs and does not directly provide IWP. CAW limit protection can be built into this design plant structure if a zero steady-state error specification is provided on the actuator error, $e_{u}$, and if this entor is observed by the controller. This approach combines the design of the nominal, linear controller and the IWP into one step and attempts to simultaneously satisfy the specification of both the nominal controller and the IWP. This approach results in a trade-off of the small perturbation, nominal, closed-loop performance for stability robustness to limit operation and may be conservative.

Reference [2] presents a general theory of antiwindup/bumpless transfer methods and uses this theory to compare the structure of several versions of the Conventional Anti-Windup (CAW) method. A version of the CAW method is demonstrated in Reference [6]. Reference [2] alludes to an IWP method denoted as the Modified Anti-Windup (MAW) method and this method was demonstrated in Reference [7] on a academic, two input, two state, two output controller. The MAW scheme uses a scalar multiplier similar to that in Reference [4], except now the scalar multiplier is applied to the controller output instead of the input. Both the CAW and the MAW schemes will be reviewed in the following.

\section{The Conventional Anti-Windup Scheme}

There are several variations on the CAW scheme as discussed in reference [2]. Figure 4 shows the structure for the version of the CAW scheme that is implemented in this paper, which includes the matrices for the nominal controller, $\left[A_{e} B_{e} ; C_{c} D_{e}\right]$, from

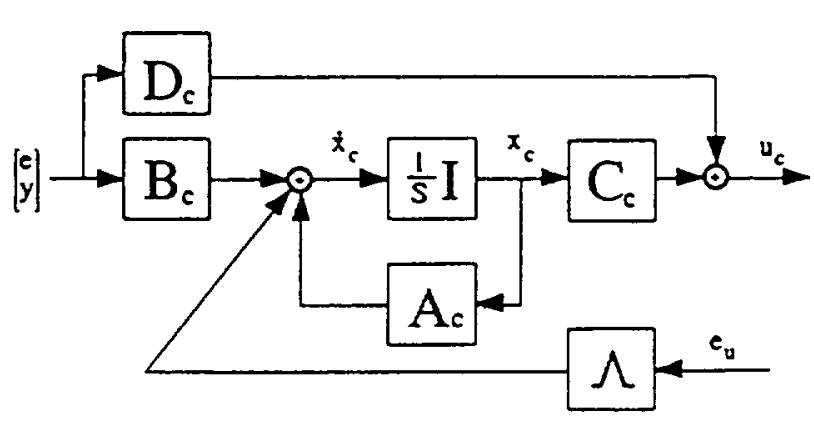

Figure 4 Implementation of the CAW Scheme

equations ( 3 ) and (4), plus an additional contribution to the controller state derivative calculation, $\Lambda e_{\mathrm{g}}$, as shown in Figure 4. This term is memoryless, since $\Lambda$ is a constant gain matrix. Using $e_{s}=u_{c}{ }_{c}-u_{c}, u_{c}$ as defined in equation (4), and the fact that $u_{c}{ }^{2}=u_{u}$ whenever the $i$ 'th actuator is limited, results in the following $\mathrm{CAW}$ controller state equation:

$$
\begin{aligned}
\dot{x}_{c} & =A_{c} x_{c}+B_{c}\left[\begin{array}{l}
e \\
y
\end{array}\right]+\Lambda L e_{u} \\
& =\left(A_{c}-\Lambda L C_{c}\right) x_{c}+\left(B_{c}-\Lambda L D_{c}\right)\left[\begin{array}{l}
e \\
y
\end{array}\right]+\Lambda L u_{1}
\end{aligned}
$$

Note in equation (5), $u_{1}$ is a vector of actuator limits. The CAW controller output equation is the same as equation (4). Note that the nominal controller is active as long as $\mathrm{L}$ is zero. $\mathrm{L}$ is a diagonal limit indicator matrix of 1 's and 0 's, one indicating a limited actuator. For the case where the third actuator is limited for a controller with 4 outputs, $L$ is defined below as:

$$
L=\left[\begin{array}{llll}
0 & 0 & 0 & 0 \\
0 & 0 & 0 & 0 \\
0 & 0 & 1 & 0 \\
0 & 0 & 0 & 0
\end{array}\right]
$$

The controller stability can be examined by studying the finite number of combinations of actuator limits using $\left(A_{c}-\Lambda \perp C_{e}\right)$. The matrix $\Lambda$ is the only design parameter for the CAW scheme. The approach taken here for designing $\Lambda$ follows the "tracking mode" implementation suggested by Aström and Wittenmark, as discussed in Reference [2]. For this implementation the nominal controller is in modal form and $\Lambda$ was designed to contribute to the diagonal of controller system matrix, $A_{r}$. This is accomplished if $\Lambda L C$ is diagonal. This is approximated using $\Lambda-\eta C^{*}$ ( \# indicates the pseudoinverse and $\eta$ is a scalar). All possible limit 
combination were then checked for controller stability. There are other methods for selecting $\Lambda$ and this explains the family of CAW schemes. The idea behind this CAW scheme is to track any actuator limit by backcalculating the controller state such that the actuator limits are satisfied.

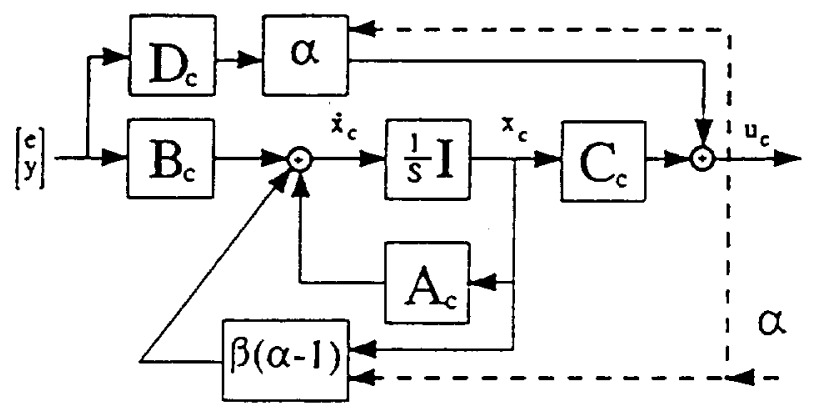

Figure 5 Implementation of the MAW Scheme

\section{The Modified Anti-Windup Scheme}

The MAW scheme provides IWP without modifying the direction of the control calculated output vector, as shown in Figure 3. Figure 5 shows the implementation structure of the MAW scheme that is used in this paper. Note that the nominal controller, as described in equations (3) and (4), is active as long as $\alpha=1$. When $\alpha \neq 1$, the nominal controller is modified by the two additional blocks shown in Figure 5. One of these blocks scales the feedthrough term $D_{c}\left[e^{T} y^{T}\right]^{T}$ by the scalar, $\alpha$. The other block "effectively" scales the $\mathrm{C}_{\mathrm{e}} \mathrm{x}_{\mathrm{c}}$ term by $\alpha$ using additional state feedback to the controller state derivative calculation using a gain of $\beta(\alpha-1) . \quad \beta$ is a constant scalar design parameter and is always greater than $0 . \alpha$ is defined as follows:

$$
\begin{aligned}
& \alpha(t)=1, \quad \text { if } u_{c i}^{L}=u_{c 1}, \quad \text { else } \\
& \alpha(t)=\min _{i}\left[\frac{u_{c}^{L}(t)_{1}}{u_{c}(t)_{1}}\right], \quad u_{c}(t)_{i} \neq 0
\end{aligned}
$$

The above ratio is the result of the division of the $i$ 'th element of output from the limit block, $u_{i}{ }^{2}$, by the i'th element of controller output, $u_{\mathrm{c}}$. Note that this is the ratio of perturbed variables and it has been assumed that $u_{c}$ and $u_{c}{ }^{2}$ are of the same numerical sign. Whenever $\left|u_{c}\right|>\left|u_{u}\right|$, for any $i$, then $\alpha$ is equal to the smallest of the ratios indicated in equation 7. This implies $0<\alpha \leq 1$. The controller equations for the MAW scheme corresponding to Figure 5 are as follows:

$$
\begin{gathered}
\dot{x}_{c}=\left(A_{c}+\beta(\alpha-1) D x_{c}+B_{c}\left[\begin{array}{l}
e \\
y
\end{array}\right]\right. \\
u_{c}=C_{c} x_{c}+\alpha D_{c}\left[\begin{array}{l}
e \\
y
\end{array}\right]
\end{gathered}
$$

The MAW structure only modifies the real part of the controller eigenvalues, regardless of the controller coordinates. This can be seen by applying a coordinate transformation matrix, $T$, to the MAW controller system matrix, $\left(A_{c}+\beta(\alpha-1) I\right)$, as shown below:

$$
T^{-1}(A+\beta(\alpha-1) I) T=T^{-1} A T+\beta(\alpha-1) \mid
$$

T does not change the scalar term and thus the MAW scheme is independent of the controller coordinates. Also, since $\beta>0$ and $(\alpha-1) \leq 0$ for $0<\alpha \leq 1$, the MAW scheme moves the real part of the eigenvalues of the nominal controller further into the left half plane by the amount $\beta(\alpha-1)$. Thus the MAW can never make the controller unstable. It would be ideal if this method guaranteed the stability of the closed-loop system, but this is an area for future study.

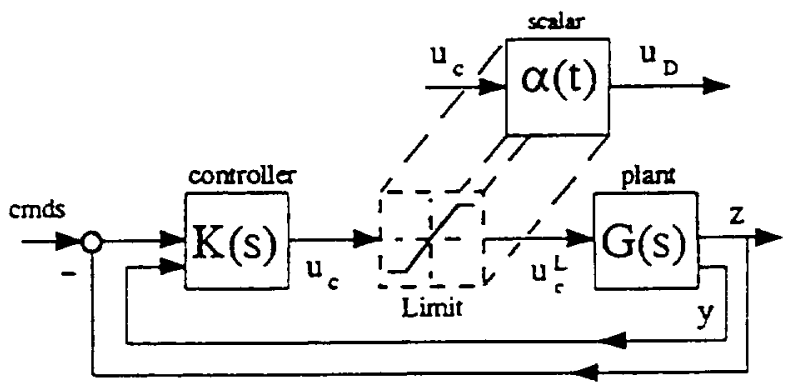

Figure 6 Idea Behind the MAW Scheme

The idea behind the MAW scheme is shown in Figure 6 by replacing the limit block with the scalar variable, $\alpha$. Note that $a$ scales all of the actuator values when any actuator is constrained. The desired control calculated output value is defined as $u_{u}=\alpha u_{c}$ where $\alpha$ is defined in equation (7). This scalar multiplier block does not provide IWP, so $a$ is moved into the control system block using block diagram manipulation as shown in Figure 7. The feedthrough term, $D_{s}\left[e^{r} y^{r}\right]^{r}$ can be scaled directly by $\alpha$, but the controller state must by modified through the controller state derivative, unless a resetable integrator is used. There are many ways to modify the controller state derivative and the following scheme was selected because it provides fast 
limit tracking and because it resembles the CAW scheme. By assigning a portion of the actuator error, $e_{a}$, to the controller state and defining the desired state vector, $x_{c d}=\alpha x_{c}$ the state error vector can now be defined as $e_{x c}=x_{c d}-x_{r}=\alpha x_{r}-x_{c}=(\alpha-1) x_{c}$. The desired output equation can now be written as follows:

$$
\begin{aligned}
u_{\alpha d} & =\alpha u_{c} \\
& =C_{c} a x_{c}+\alpha D_{c}\left[\begin{array}{l}
e \\
y
\end{array}\right] \\
& =C_{c} x_{\alpha d}+\alpha D_{c}\left[\begin{array}{l}
e \\
y
\end{array}\right]
\end{aligned}
$$

A proportional gain controller acting on the state error vector can be used to drive $x_{c}$ to $x_{c d}$. Figure 5 shows the resulting structure with $\beta$ as the proportional gain. It is interesting to note that the MAW scheme is independent of the controller matrices and thus would not have to be scheduled if the controller were gain scheduled.
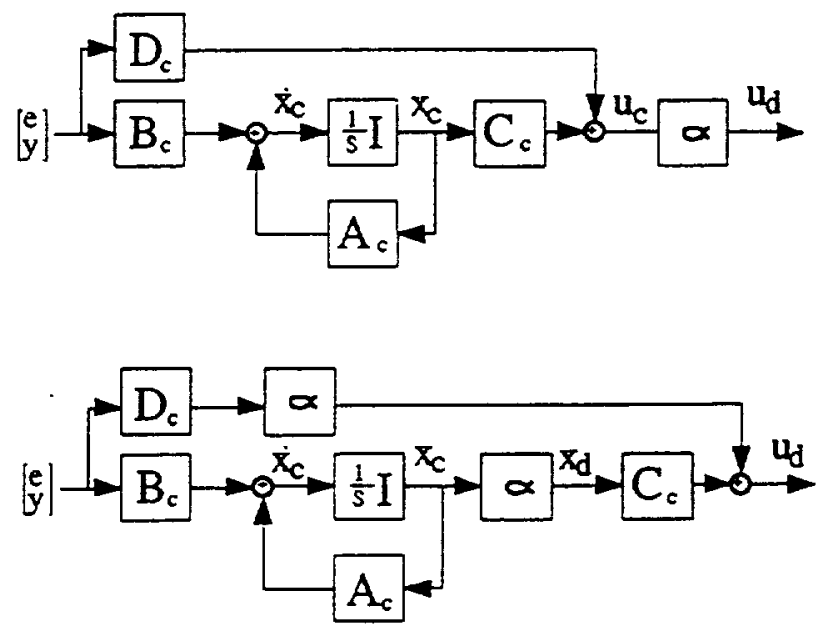

Figure 7 Obtaining IWP for the MAW Scheme via Block Diagram Manipulation

\section{Turbofan Engine Limit Model}

Reference [6] discusses the implementation and response of a closed-loop engine control system as part of the evaluation of an integrated flight/propulsion control system. A linear version of the closed-loop propulsion control system and plant used in Reference [6] is used in the following example. Figure 8 shows the closed-loop system, comprised of a nominal controller with IWP, linear actuator models, actuator limits, and a linear engine model in the form given by

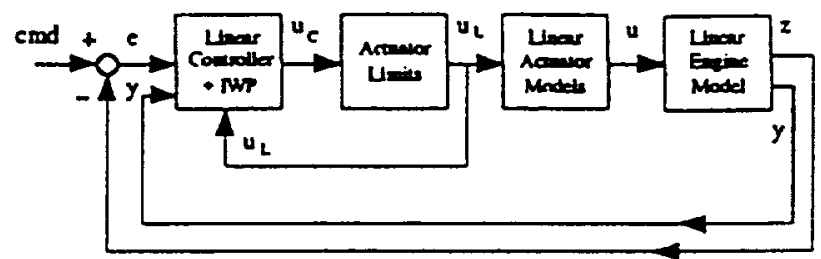

Figure 8 Closed-loop Turbofan Engine Example

equations (1) and (2). The inputs, controlled variables, extra feedback, and state are defined as follows:

$$
\begin{aligned}
& \mathbf{u}=[\mathbf{W F} 36, \text { A8, Eta, A78 }]^{\mathrm{T}} \\
& z=[\mathrm{N} 2, \mathrm{FG}, \text { FGE, FGV }]^{\mathrm{T}} \\
& \mathbf{y}=\mathbf{N} 2 \\
& \mathbf{x}=[\mathrm{N} 2, \mathrm{~N} 25]^{\mathrm{T}}
\end{aligned}
$$

The above variables are defined in the nomenclature list. The limits for this example are hard actuator limits reflected onto the linear perturbation variables and these limits were further reduced to study the limit operations of the IWP schemes. For example, at the design point for this linear engine model, the nominal value for the ejector butterfly valve angle, Eta, was 64 degrees and the maximum butterfly valve angle is 90 degrees (full open). This yields a perturbation limit of 26 degrees for Eta, but the perturbation limit was artificially lowered to 9 degrees to study the limit operation of the two IWP schemes. Other perturbation limits were similarly modified during this study. The nominal control system design is a linear, perturbation design as discussed in Reference [8]. The engine controller is 7 th order. The reason for this relatively high order controller, (the plant is second order), is that the original controller was designed with a higher order model of the engine which included temperature and pressure state variables. The eigenvalues for the open-loop engine, the nominal controller and the controller with MAW active are shown in Table 1. The MAW controller eigenvalues were calculated using $\beta=10$ and $\alpha=0.9$ for this example but $\alpha$ variations in the range from 0.8 to 1.0 were observed in the simulations. The MAW scheme makes the real part of all the eigenvalues more negative by the amount $\beta(\alpha-1)$, as seen in Table 1. Table 2 shows the controller eigenvalues with the $\mathrm{CAW}$ scheme for various combinations of actuator limits. For example, the marrix $L=\operatorname{diag}\left[\begin{array}{llll}0 & 0 & 1 & 0\end{array}\right]$ corresponds to the case with the ejector butterfly valve, Eta, is limited. $\eta=10$ is used in this example to provide reasonable limit tracking. The closed-loop results for the CAW and MAW controller schemes will now be examined. 


\section{Results of the Turbolan Engine Example}

Various commands were simulated for the closedloop system using both IWP schemes and the results were compared. One response that gives a good summary of the results will be examined. The command is an ejector thrust command, FGE command of FGE alone is atypical, but it allows the changes in coupling to be more easily seen since the other loops are trying to regulate to zero. This single command does not detract from the comparison of the

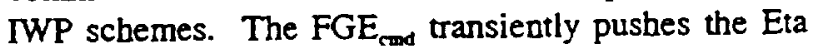
actuator into the limit. Figure 9 shows the controller outputs, $u$, for the three cases: (1) the Modified AntiWindup (MAW) scheme; (2) the Conventional AntiWindup scheme; (3) the unlimited case. Note that ETA
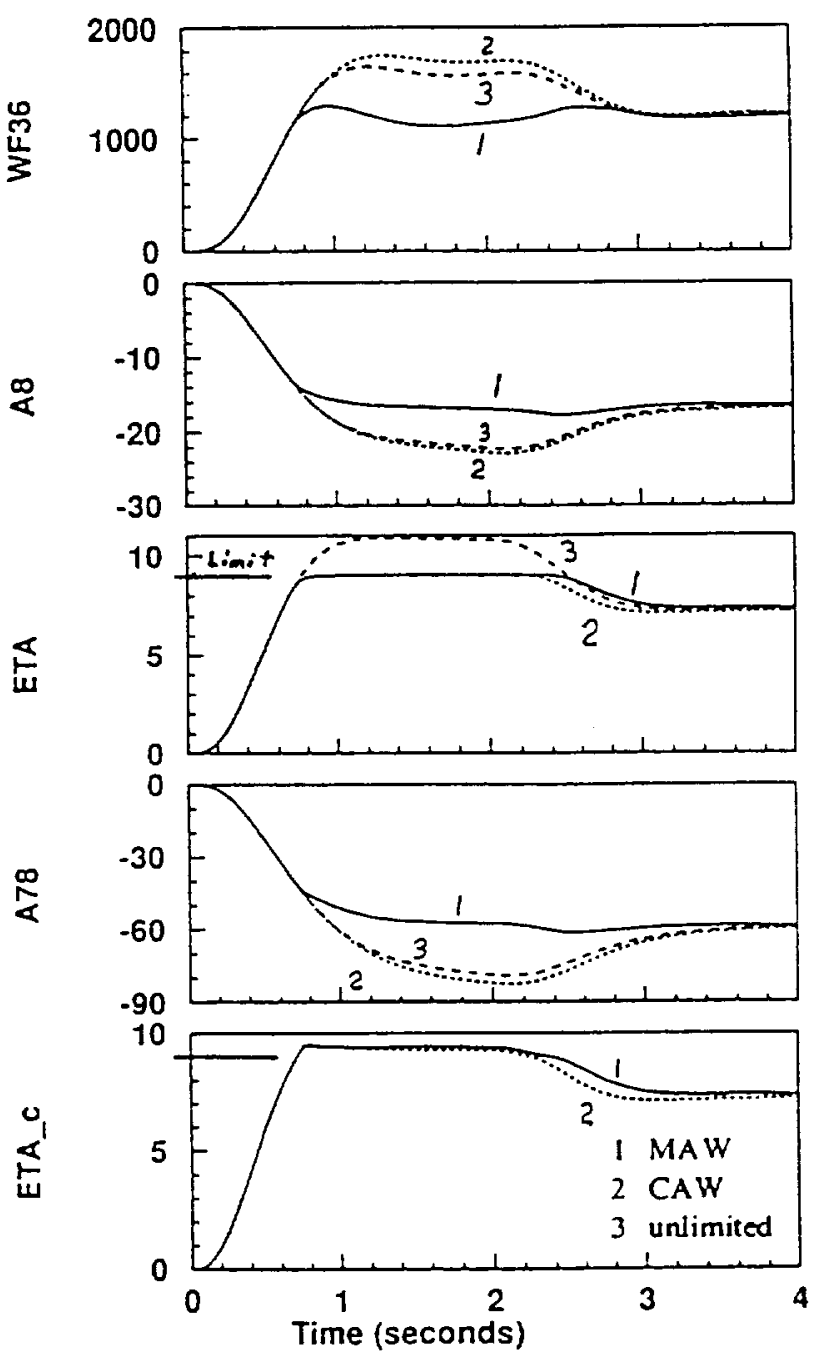

Figure 9 Comparison of Actuator Responses equal to nine is the only limit encountered. The last plot of Figure 9, the controller output value, ETA_c, shows that both IWP schemes closely tracks the limit value. The response for WF36, A8, and A78 in the CAW case are similar to the response for the unlimited case, but for the MAW scheme, all the actuators are all scaled back by $\alpha$. Figure 10 compares the closed-loop system outputs. FGE command is also shown in the third plot of Figure 10 for reference. Note that the level of coupling for the CAW scheme appears to be slightly greater that the coupling for the case with the MAW scheme. However, the FGE command tracking for the controller with CAW appears to be better than the command tracking for the controller with the MAW scheme. $\alpha$ is shown in Figure 10 for reference.
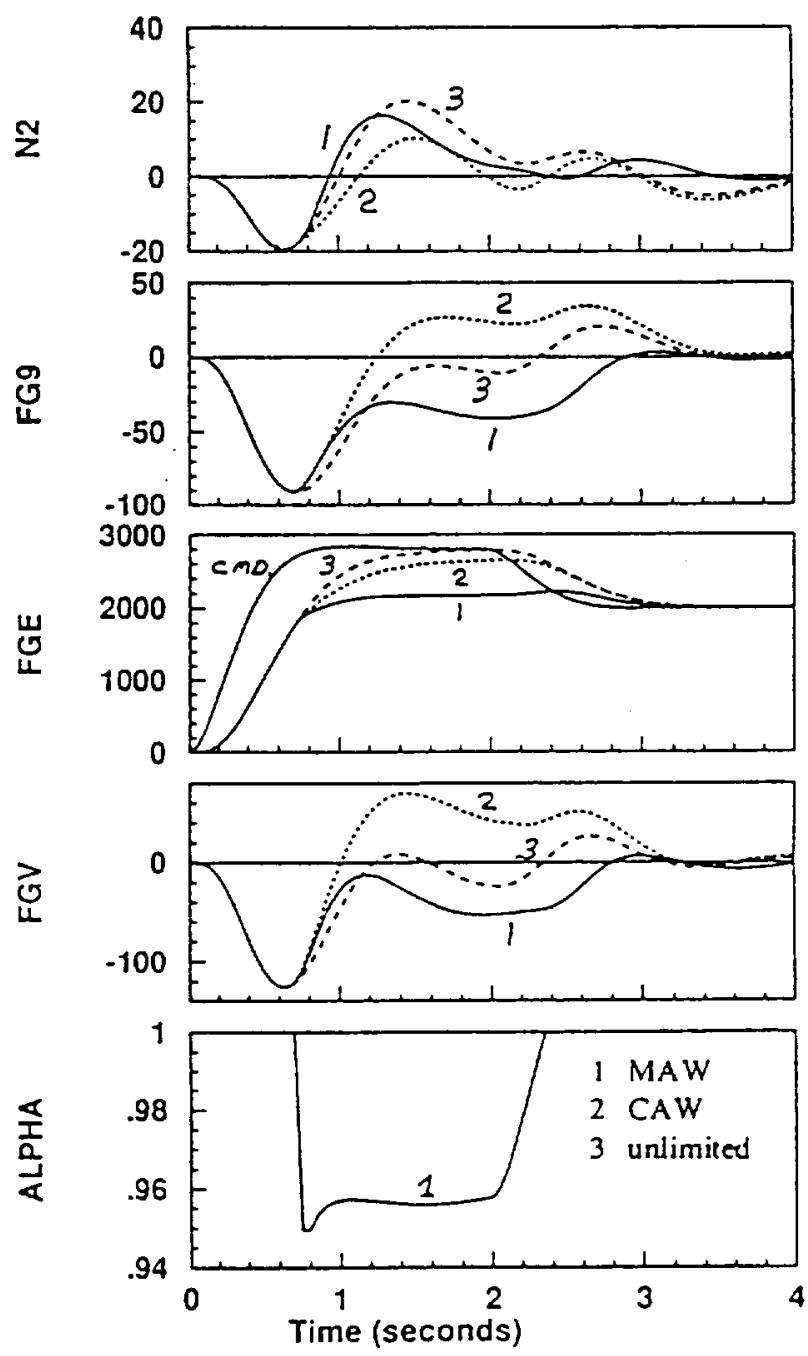

Figure 10 Comparison of Closed-Loop Outputs 
From Figure 9 and Figure 10 it is difficult to determine if one method is superior to the other and that was fairly typical of all the responses that were examined. This turbofan engine example does not reveal a clearly superior method, perhaps because this example is not particularly sensitive to changes in the direction of the control vector. Another example that does exhibit sensitivity to the direction of the control vector is the aircraft pitch control problem. In the STOVL aircraft example currently being studied [6], FGE and FGV from the current turbofan engine example balance the pitching moment to provide pitch control for a STOVL aircraft. Preliminary results from this study indicate a distinct advantage for the MAW over the CAW scheme and these results will be published in the future.

One claim of the scalar, MAW scheme is that it does not modify the direction of the input vector. This needs clarification. Multiplying a vector by $\alpha\left(b_{b}\right)$ does not change the vector direction at that time, $t_{0}$. This does not mean the vector direction of the limited system with MAW will be a scalar multiple of the unlimited closed-loop system. For the vector direction, relative to the unlimited case, to be maintained exactly, the zero crossings for the unlimited and the MAW schemes would have to be identical. To probe this directionality question further, consider the constant value of $\alpha$ shown in Figure 11a. In this case, the constant $\alpha$ scales the actuator and the inverse of $\alpha$ scales the corresponding measurements. This has the effect of fictitiously making the plant look larger than it is to the controller, $(y / \alpha, 0<\alpha<1)$. Time history responses of this closed loop system for various constant values of $\alpha$ are all properly scaled and the zero crossings of the controller outputs and plant outputs are identical to the case for $\alpha=1$. In fact, block diagram manipulation shows that for $\alpha=$ constant, this is really just a scalar reduction of the command as shown in Figure $11 \mathrm{~b},(1 / \alpha \mathrm{K}(\mathrm{s}) \alpha=$ $\mathrm{K}(\mathrm{s})$ ). Consider a time varying value for $\alpha, \alpha(\mathrm{t})$, in Figure 11a. For this case, directionality relative to the case where $\alpha=$ constant, is not maintained. The reason for this is that the plant has memory and the plant state vector does not get appropriately scaled by a time varying scalar. But Figure $1 \mathrm{lb}$ does help to understand how the MAW works. The MAW scheme will appear as a variabie rate limit on all the commands. There still remains a question regarding the affect the MAW has on the closed-loop feedback properties.

Several practical aspects of CAW and the MAW schemes need to be addressed. One of the advantages of the MAW scheme is that it does not have to be altered if the controller is gain scheduled. This is an advantage over the CAW scheme where the pseudoinverse of the $C_{r}$ matrix would have to be calculated online as it was scheduled throughout the operating range. One disadvantage of the MAW scheme is that it is implemented on the perturbation variables. This requires that the numeric signs of $u_{c}$ and $\mathrm{u}_{\mathrm{e}}{ }^{\mathrm{L}}$ must be the same, which may not always be true depending on the cause of the actuator limit. For example engine safety limits like the fan surge margin may have internal logic that makes it possible to have the perturbed variables $u_{c}{ }^{l}$ and $u_{c}$ of different numeric signs. Additional logic would be required to handle these special cases. The CAW scheme is based on the actuator error, $e_{a}$, and does not have this problem. In terms of stability, it is desired to have a test for closedloop stability. While this is not yet available, the MAW scheme can not make the controller unstable, but on the other hand the CAW scheme has a finite number of cases to test for closed-loop stability.

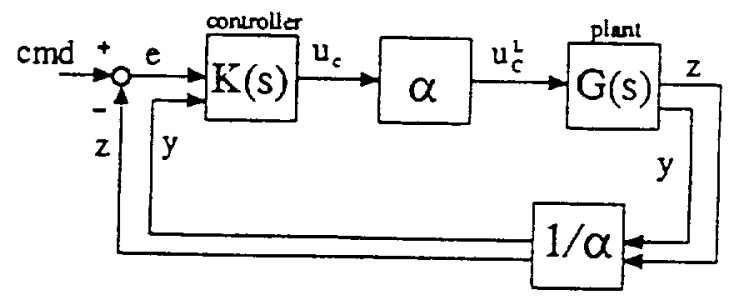

Figure 1la Scaling the Plant

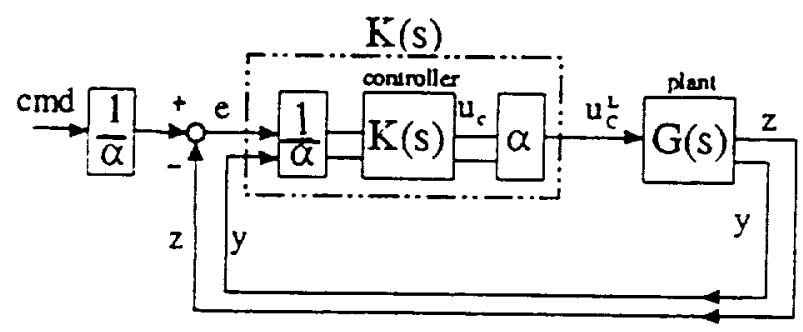

Figure $11 \mathrm{~b}$ Scalar Modification of Commands 


\section{Conclusions}

The conventional anti-windup (CAW) and the modified anti-windup (MAW), nulti-variable limit and integrator windup protection (IWP) schemes were described and compared. A design example consisting of a model of a turbofan engine control system was presented to compare the closed-loop limit operation of the two IWP schemes. The example system presented does not exhibit sensitivity to changes in the direction of the control vector and thus neither of the IWP schemes were shown to be clearly superior. Both schemes were successful in providing integrator windup protection and limit tracking. Nevertheless, the MAW does look promising as it attempts to maintain the loop properties by moving the limit problem out of the feedback loop and on to the commands as rate limits. An example system that does exhibit sensitivity to changes in the direction of the control vector, the pitch control of a STOVL aircraft, is currently being investigated.

\section{Acknowledgements}

The author would like to thank the following for their helpful suggestions and discussions: Vincent Marcopoli and Steve Phillips from Case Western Reserve University, Electrical Engineering and Applied Physics Dept. and Jeff Musgrave and Sanjay Garg from the Advanced Control Technology Branch, NASA Lewis Research Center.

\section{References}

[1] Garg S., Ouzts P.J., Lorenzo C.F., Mattern D., "IMPAC - An Integrated Methodology for Propulsion and Airframe Control", 1991 American Control Conference, Boston MA, NASA TM 103805.

[2] Campo P.J., Morari M., Nett C.N., "Multivariable Anti-Windup and Bumpless Transfer: A General Theory", 1989 American Controls Conference, Pittsburgh, PA. TP9-5:00, p. 1706.

[3] Wittenmark B., "Integrators, Nonlinearities, and Anti-reset Windup for Different Control Structures", 1989 American Controls Conference, Pittsburgh, PA, TP9-2:30, p. 1679.
[4] Kapasouris P., Athans M., Stein G.. "Design of Feedback Control Systems for Stable Plants with Saturating Actuators", 27th Conference on Decision and Control, 1987, WP2-5:00, p 469.

[5] Boyd S.P., Barratt C.H., "Linear Controller Design, Limits of Performance", Prentice Hall, 1991, p230, 247.

[6] Mattern D., Garg S., "Propulsion System Performance Resulting from an Integrated Flight/Propulsion Control Design", NASA TM 105874, presented at the 1992 AJAA Guidance, Navigation and Control Conference.

[7] Doyle J.C., Smith R.S., and Enns D.F., "Control of plants with input saturation nonlinearities. Proceedings of the 1987 American Control Conference, p 1034, 1987.

[8] Garg S., D.Mattem,"Application of an Integrated Flight/Propulsion Control Design Methodology to a STOVL Airctaft", AIAA-91-2792, presented at the 1991 AlAA Guidance, Navigation and Control Conference, NASA TM 105254.

\begin{tabular}{|ccc|}
\hline $\begin{array}{c}\text { Table } 1 \\
\text { open-loop } \\
\text { Plant }\end{array}$ & $\begin{array}{c}\text { Eigenvalues for the } \\
\text { Nominal }\end{array}$ \\
controller & $\begin{array}{c}\text { controller } \\
\text { with MAW }\end{array}$ \\
\hline-2.97 & -0.004 & -1.004 \\
-6.33 & -0.007 & -1.007 \\
& -0.011 & -1.011 \\
& -0.011 & -1.011 \\
& -0.011 & -1.011 \\
& -0.171 & -1.171 \\
& -2.896 & -3.896 \\
\hline
\end{tabular}

\begin{tabular}{|cccc|}
\hline \multicolumn{5}{|c|}{ Table 2} \\
\hline Elgenvalues for CAW scheme \\
\hline - $[0100]$ & {$[0111]$} & {$[1010]$} & {$[1111]$} \\
\hline-0.004 & -0.004 & -0.004 & -0.004 \\
-0.008 & -0.009 & -0.010 & -0.010 \\
-0.011 & -0.150 & -0.011 & -2.285 \\
-0.011 & -2.260 & -0.168 & -20.01 \\
-0.011 & -20.01 & -2.781 & -20.01 \\
-2.801 & -20.01 & -20.01 & -20.01 \\
-20.11 & -20.64 & -20.12 & -20.64 \\
\hline L - [0100] 18 a diagonal matrix \\
with 0, 1, 0, 0 on the diagonal
\end{tabular}


Public reporting burden for this collection of intormation is estimated to average 1 hour per response, including the time for reviewing instructions, searching existing data sources, collection of information, including suggestions for reducing and reviewing the collection of information. Send comments regarding this burden estimate or any other aspect of this Davis Highway, Suite 1204. Aring suggestons lor reducing this burden, to Washington Headquarters Services, Directorate for Information Operations and Reports, 1215 Jefferson

Davis Highway, Suite 1204. Arlington, VA 22202-4302, and to the Office of Management and Budgel, Paperwork Reduction Project (0704-0188), Washington, DC 20503.

\begin{tabular}{|l|l|c|}
\hline 1. AGENCY USE ONLY (Leave blank) & $\begin{array}{c}\text { 2. REPORT DATE } \\
\text { December } 1993\end{array}$ & $\begin{array}{c}\text { 3. REPORT TYPE AND DATES COVERED } \\
\text { Final Contractor Repor }\end{array}$
\end{tabular}

4. TITLE AND SUBTITLE

Final Contractor Report

A Comparison of Two Multi-Variable Integrator Windup Protection Schemes
6. AUTHOR(S)

Duane Mattern

7. PERFORMING ORGANIZATION NAME(S) AND ADDRESS(ES)

Sverdrup Technology, Inc.

Lewis Research Center Group

2001 Aerospace Parkway

Brook Park, Ohio 44142
5. FUNDING NUMBERS

WU-505-62-50

C-NAS3-25266

B. PERForming ORGANIZATION REPORT NUMBER

E-8283

10. SPONSORINGMONITORING AGENCY REPORT NUMBER

National Aeronautics and Space Administration

Lewis Research Center

Cleveland, Ohio 44135-3191

NASA CR-194436

AIAA-93-3812

\section{SUPPLEMENTARY NOTES}

Prepared for the 1993 AIAA Guidance, Navigation, and Control Conference sponsored by the American Institute of Aeronautics and Astronautics, Monterey, California, August 9-11, 1993. Project Manager, Walter C. Merrill, Instrumentation and Control Technology Division, (216) 433-6328.

12a. DISTRIBUTIONAAVAILABILITY STATEMENT

12b. DISTRIBUTION CODE

Unclassified - Unlimited

Subject Categories 08 and 31

\section{ABSTRACT (Maximum 200 words)}

Two methods are examined for limit and integrator wind-up protection for multi-input, multi-output linear controllers subject to actuator constraints. The methods begin with an existing linear controller that satisfies the specifications for the nominal, small perturbation, linear model of the plant. The controllers are formulated to include an additional contribution to the state derivative calculations. The first method to be examined is the multi-variable version of the single-input, single-output, high gain, Conventional Anti-Windup (CAW) scheme. Except for the actuator limits, the CAW scheme is linear. The second scheme to be examined, denoted the Modified Anti-Windup (MAW) scheme, uses a scalar to modify the magnitude of the controller output vector while maintaining the vector direction. The calculation of the scalar modifier is a nonlinear function of the controller outputs and the actuator limits. In both cases the constrained actuator is tracked. These two integrator windup protection methods are demonstrated on a turbofan engine control system with five measurements, four control variables, and four actuators. The closed-loop responses of the two schemes are compared and contrasted during limit operation. The issue of maintaining the direction of the controller output vector using the Modified Anti-Windup scheme is discussed and the advantages and disadvantages of both of the IWP methods are presented.

14. SUBJECT TERMS

Controls; Turbofan engine; Limit operation 15. NUMBER OF PAGES

11

16. PRICE CODE

$\mathrm{A} 03$

\begin{tabular}{|c|c|c|}
\hline $\begin{array}{c}\text { 17. SECURITY CLASSIFICATION } \\
\text { OF REPORT } \\
\text { Unclassified }\end{array}$ & $\begin{array}{c}\text { 18. SECURITY CLASSIFICATION } \\
\text { OF THIS PAGE } \\
\text { Unclassified }\end{array}$ & $\begin{array}{c}\text { 19. SECURITY CLASSIFICATION } \\
\text { OF ABSTRACT } \\
\text { Unclassified }\end{array}$ \\
\hline
\end{tabular}

